\title{
HIF-1 mediates metabolic responses to intratumoral hypoxia and oncogenic mutations
}

\author{
Gregg L. Semenza
}

Vascular Program, Institute for Cell Engineering; Departments of Pediatrics, Medicine, Oncology, Radiation Oncology, and Biological Chemistry; and McKusick-Nathans Institute of Genetic Medicine, Johns Hopkins University School of Medicine, Baltimore, Maryland, USA.

\begin{abstract}
Hypoxia occurs frequently in human cancers and induces adaptive changes in cell metabolism that include a switch from oxidative phosphorylation to glycolysis, increased glycogen synthesis, and a switch from glucose to glutamine as the major substrate for fatty acid synthesis. This broad metabolic reprogramming is coordinated at the transcriptional level by HIF-1, which functions as a master regulator to balance oxygen supply and demand. HIF-1 is also activated in cancer cells by tumor suppressor (e.g., VHL) loss of function and oncogene gain of function (leading to PI3K/AKT/mTOR activity) and mediates metabolic alterations that drive cancer progression and resistance to therapy. Inhibitors of HIF-1 or metabolic enzymes may impair the metabolic flexibility of cancer cells and make them more sensitive to anticancer drugs.
\end{abstract}

\section{Introduction}

All human cells require a constant supply of $\mathrm{O}_{2}$ to carry out oxidative phosphorylation in the mitochondria for ATP generation. Under hypoxic conditions when $\mathrm{O}_{2}$ availability is reduced, cells generally respond in three ways: (a) cell proliferation is inhibited to prevent any further increase in the number of $\mathrm{O}_{2}$-consuming cells; (b) the rate of oxidative phosphorylation is decreased and the rate of glycolysis is increased in order to decrease $\mathrm{O}_{2}$ consumption per cell; and (c) the production of angiogenic factors is increased in order to increase $\mathrm{O}_{2}$ delivery. Mutations in cancer cells dysregulate cell growth and metabolism, but the mechanisms and consequences of this dysregulation vary widely from one cancer to another and even one from cancer cell to another. In some cancer cells, $\mathrm{O}_{2}$ still regulates the rate of cell proliferation, whereas others continue to divide even under severely hypoxic conditions; some cancers are well vascularized and perfused, whereas most cancers contain steep $\mathrm{O}_{2}$ gradients that reflect the distance to the nearest blood vessel, the number of intervening cells and their metabolic activity, and the rate at which blood is flowing through the vessel. The metabolism of individual cancer cells reflects the presence of particular genetic alterations, which may alter metabolism in an $\mathrm{O}_{2}$-independent manner, as well as the spatial and temporal heterogeneity of $\mathrm{O}_{2}$ availability within the tumor microenvironment. This Review summarizes the role of HIF-1 in the regulation of cancer cell metabolism, focusing primarily on the use of glucose as a metabolic substrate.

\section{HIF-1 mediates adaptive responses to reduced $\mathrm{O}_{2}$ availability}

HIF-1 is a heterodimer, consisting of an $\mathrm{O}_{2}$-regulated HIF- $1 \alpha$ subunit and a constitutively expressed HIF- $1 \beta$ subunit $(1,2)$, that binds to the consensus sequence $5^{\prime}$-RCGTG- $3^{\prime}$ that is present within or near HIF-1-regulated genes (3). HIF- $1 \alpha$ protein stability is negatively regulated by $\mathrm{O}_{2}$-dependent prolyl hydroxylation (Figure 1), which enables binding of the von Hippel-Lindau tumor suppressor protein (VHL), the recognition subunit of an E3 ubiquitin ligase that ubiquitylates HIF- $1 \alpha$, thereby targeting it for proteasomal degradation (4). HIF-1 $\alpha$ stability is also modulated according to

Conflict of interest: The author has declared that no conflict of interest exists. Citation for this article: J Clin Invest. 2013;123(9):3664-3671. doi:10.1172/JCI67230. cellular metabolic status because, in addition to $\mathrm{O}_{2}$, the TCA cycle intermediate $\alpha$-ketoglutarate is also a reaction substrate for prolyl hydroxylases. The hydroxylases insert one oxygen atom into a proline residue (either Pro-403 or Pro-564 in human HIF-1 $\alpha$ ), and the other oxygen atom is inserted into $\alpha$-ketoglutarate, splitting it into succinate and $\mathrm{CO}_{2}$.

Database searches using the HIF- $1 \alpha$ sequence identified HIF- $2 \alpha$, which is also $\mathrm{O}_{2}$-regulated, dimerizes with $\mathrm{HIF}-1 \beta$, and activates gene transcription $(5,6)$. HIF-1 $\alpha$ homologs have been identified in all metazoan species analyzed and are expressed in all cell types, whereas HIF- $2 \alpha$ homologs are only found in vertebrates and are expressed in a restricted number of cell types $(7,8)$, although many cancer cells express both HIF- $1 \alpha$ and HIF- $2 \alpha(9,10)$. Because the battery of genes that is activated by HIF-1 and HIF-2 in response to hypoxia is unique within each cell, the number of HIF target genes, which currently exceeds 1,000 , continues to increase as new cell types are analyzed by ChIP techniques such as ChIP-chip $(11,12)$ and ChIP-seq (13).

Many cancers contain areas of intratumoral hypoxia, and primary tumors with low oxygenation $\left(\mathrm{PO}_{2}<10 \mathrm{mmHg}\right)$ are associated with an increased risk of metastasis and patient mortality (14). Increased HIF-1 $\alpha$ levels are also associated with increased risk of mortality in many human cancers, including those of the bladder, brain, breast, colon, esophagus, head/neck/oropharynx, liver, lung, pancreas, skin, stomach, and uterus, as well as in acute lymphocytic and myeloid leukemias (15).

In some cancers, such as renal clear cell carcinoma, clinical data indicate that HIF- $2 \alpha$ overexpression is associated with disease progression and mortality, whereas HIF-1 $\alpha$ expression is silenced, often by gene deletion (16). In contrast, in colon carcinoma, clinical data indicate that HIF- $1 \alpha$ overexpression is associated with disease progression and HIF- $2 \alpha$ expression is silenced (17). At the molecular level, there is also heterogeneity. In most cell types, genes have been identified that are transactivated (a) only by HIF-1 $\alpha$, such as lactate dehydrogenase A (LDHA) and other glycolytic enzyme genes; (b) only by HIF-2 $\alpha$, such as TGFA; or (c) by both HIF- $1 \alpha$ and HIF- $2 \alpha$, such as VEGF (18). Yet in MCF-7 breast cancer cells, although binding of both HIF- $1 \alpha$ and HIF- $2 \alpha$ to HIF target genes was demonstrable by ChIP, only knockdown of HIF-1 $\alpha$ resulted in decreased gene expression (19). In contrast, in renal carcinoma cell lines, in 


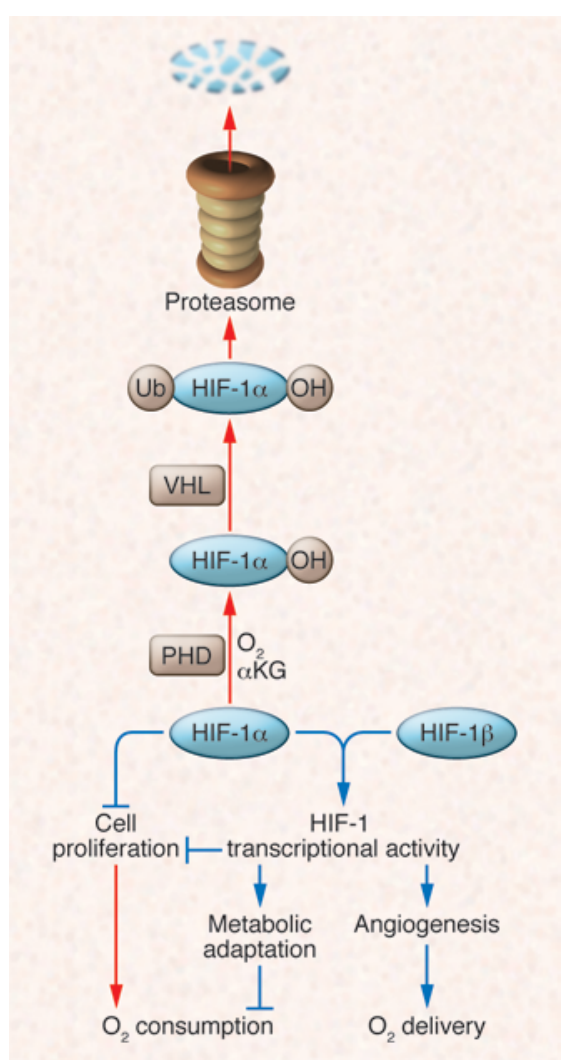

which HIF-2 $\alpha$ but not HIF- $1 \alpha$ is expressed, all HIF target genes (including glycolytic enzyme genes) are expressed in a HIF- $2 \alpha-$ dependent manner (20). This cell type-specific HIF- $\alpha$ subunit target gene selectivity may be due to the regulated recruitment of as-yet-unidentified coactivators or corepressors.

HIF- $1 \alpha$ contributes to the regulation of multiple adaptive responses to hypoxia, including cell proliferation, metabolism, and angiogenesis. The regulation of angiogenesis and metabolism are mediated via the transcriptional activity of the HIF- $1 \alpha / \mathrm{HIF}-1 \beta$ heterodimer, whereas the regulation of cell proliferation is mediated through both transcriptional and nontranscriptional effects of HIF-1 $\alpha$ (Figure 1).

Angiogenesis. HIF-1 activates the transcription of VEGF (21) and many other genes encoding angiogenic cytokines and growth factors in hypoxic cells, which stimulate angiogenesis and vascular remodeling that lead to improved tissue perfusion and increased $\mathrm{O}_{2}$ delivery in normal tissues (22). However, in many cancers, the vascular response is dysregulated, such that the blood vessels are structurally and functionally abnormal, leading to persistent defects in perfusion and oxygenation (23). Both the abnormal tumor vessels and the resulting intratumoral hypoxia that these vessels perpetuate contribute to metastasis, the process by which most cancers kill their hosts, and HIF-1 activates the transcription of genes that control multiple steps in the metastatic process (24).

Proliferation. HIF-1 $\alpha$ mediates G1 cell cycle arrest by transcriptional or nontranscriptional mechanisms in different cell types. Several transcriptional mechanisms have been reported: (a) HIF$1 \alpha$ may displace MYC from the promoter of CDKN1A gene, which

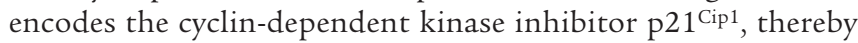
de-repressing its transcription (25); (b) HIF-1 $\alpha$ may interact with

\section{Figure 1}

HIF-1 regulates the balance between $\mathrm{O}_{2}$ supply and demand. In well-oxygenated cells, prolyl hydroxylase domain (PHD) proteins use $\mathrm{O}_{2}$ and $\alpha$-ketoglutarate $(\alpha \mathrm{KG})$ to hydroxylate $\mathrm{HIF}-1 \alpha$, which is then bound by VHL, ubiquitylated, and degraded by the proteasome. Under hypoxic conditions, the hydroxylation reaction is inhibited and HIF- $1 \alpha$ accumulates and regulates cell proliferation directly or dimerizes with HIF-1 $\beta$ to activate the transcription of hundreds of target genes, many of which encode enzymes and transporters that control cell metabolism. Red and blue arrows indicate reactions that are favored in aerobic and hypoxic conditions, respectively.

$\beta$-catenin and block $\beta$-catenin-dependent MYC transcription (26); and (c) HIF-1 may inhibit MYC activity by activating transcription of the MXI1 gene, which encodes a MYC repressor (27). More recently, HIF-1 $\alpha$ was shown to bind directly to components of the prereplicative complexes that assemble at origins of replication and inhibit activation of the minichromosome maintenance helicase, thereby directly blocking DNA replication by a mechanism that is independent of transcription (28).

\section{HIF-1 reprograms the metabolism of hypoxic cells}

Reduced $\mathrm{O}_{2}$ availability has profound effects on cellular metabolism. Glucose and glutamine are considered the primary metabolic substrates of cancer cells, and their utilization is markedly changed in response to hypoxia, as described below.

Glucose catabolism and energy metabolism. Because HIF-1 is present even in simple metazoan species with no specialized systems for $\mathrm{O}_{2}$ delivery, it is likely that the primordial role of HIF-1 was to regulate $\mathrm{O}_{2}$ consumption by orchestrating the switch from oxidative to glycolytic metabolism under hypoxic conditions. Analysis of $\mathrm{Hifl}^{-/-}$mouse embryonic stem cells revealed that expression of genes encoding glucose transporters and virtually all of the glycolytic enzymes were induced by hypoxia and/or regulated by HIF-1 (29). The consequences of HIF-1 $\alpha$ deficiency are particularly dramatic in mouse embryonic fibroblasts (MEFs): when cultured in the presence of $1 \% \mathrm{O}_{2}$, most of these cells die within 96 hours, whereas wild-type cells continue to proliferate, albeit at reduced rates compared with the standard tissue culture conditions (30, 31). Conventional wisdom holds that cells switch from oxidative to glycolytic metabolism under hypoxic conditions to maintain ATP production, but $1 \% \mathrm{O}_{2}$ was not limiting for ATP production in Hif1 $a^{-/-}$MEFs, which had higher ATP levels than wild-type cells (31). Rather than dying of ATP depletion, Hifla $a^{-/}$MEFs die from excess production of ROS, which results from the failure to switch from oxidative to glycolytic metabolism $(30,31)$.

Hypoxic MEFs reprogram glucose metabolism through transactivation of the pyruvate dehydrogenase (PDH) kinase, isozyme $1(P d k 1)$ and the BCL2/adenovirus E1B 19-kDa interacting protein 3 (Bnip3) genes by HIF-1 (30-32). PDK1 phosphorylates and inactivates PDH, thereby inhibiting the conversion of pyruvate to acetyl-CoA $(\mathrm{acCoA})$ for entry into the TCA cycle (Figure 2A). HIF-1 also activates expression of $L d$ ha, which converts pyruvate to lactate, the terminal glycolytic product $(3,29)$. Bnip3 encodes a protein that promotes mitochondrial-selective autophagy as a means to reduce oxidative metabolism (31). The increased activities of BNIP3, PDK1, and LDHA dramatically alter the fate of pyruvate in hypoxic cells. Expression of the BNIP3 and PDK1 homologs BNIP3L and PDK3, respectively, is also induced by hypoxia in a HIF-dependent manner and contributes to mitochondrial autophagy and metabolic reprogramming in cancer cells (33-35). 
A

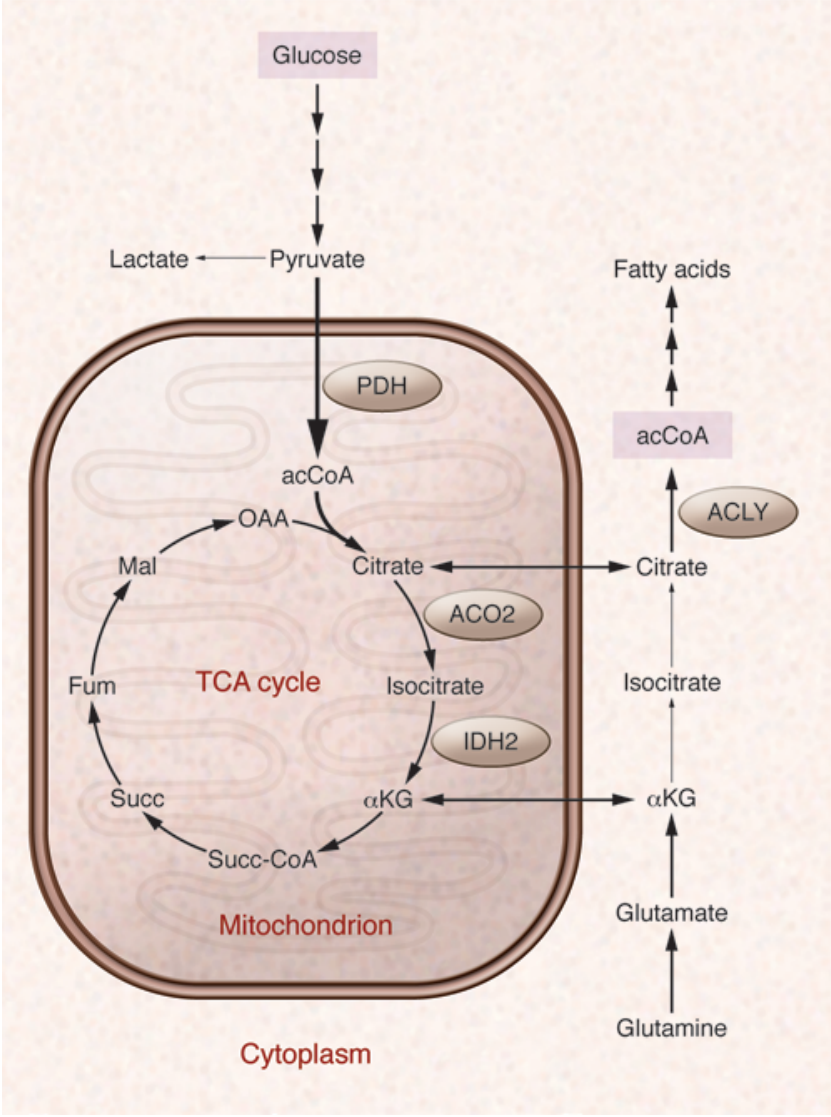

B

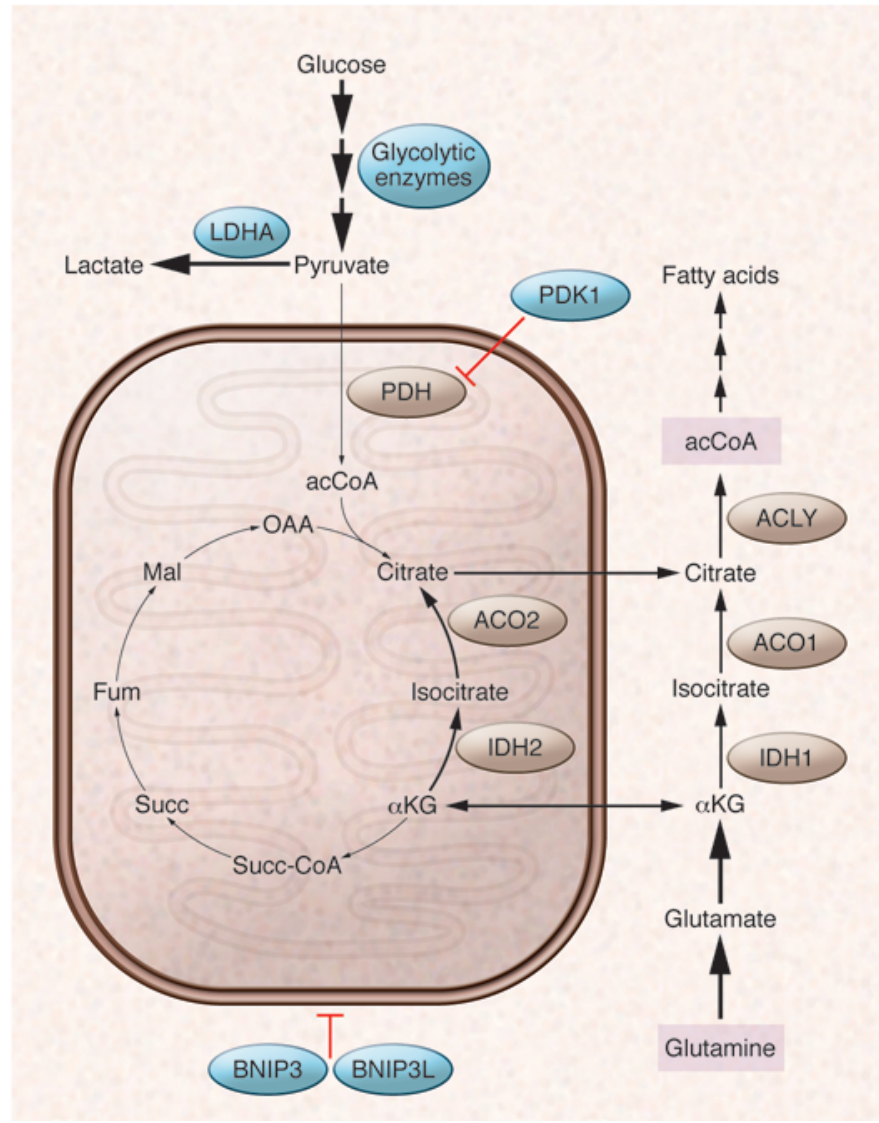

Figure 2

Oxygen-dependent regulation of glucose and glutamine metabolism by HIF-1. (A) In well-oxygenated cells, glucose is metabolized to pyruvate, which is converted to acCoA by PDH for entry into the TCA cycle. Glucose-derived citrate is shuttled to the cytosol and converted to acCoA by ATP-citrate lyase (ACLY) for fatty acid synthesis. Glutamine is converted to $\alpha$ KG for entry into the TCA cycle. (B) In hypoxic cells, HIF-1 activates the transcription of several genes: (a) PDK1 and genes encoding other isoforms of PDH kinase, which phosphorylates and inactivates PDH; (b) LDHA, which converts pyruvate to lactate; (c) other glycolytic enzymes and glucose transporters to increase flux through the glycolytic pathway; and (d) BNIP3 and BNIP3L, which trigger mitochondrial selective autophagy. To compensate for the reduced flux of glucose to citrate, reductive carboxylation of glutamine generates cytosolic citrate for fatty acid synthesis. Note the change in direction of the reactions catalyzed by aconitase (ACO1, ACO2) and isocitrate dehydrogenase (IDH1, IDH2) under hypoxic conditions. Blue circles indicate proteins that are products of HIF-1 target genes. Fum, fumarate; Mal, malate; OAA, oxaloacetate; Succ, succinate; Succ-CoA, succinyl coenzyme A.

Forced expression of PDK1 or BNIP3 was sufficient to rescue Hifla $a^{-/-}$MEFs from ROS-mediated cell death under conditions of prolonged hypoxia $(30,31)$. Whereas ROS is associated with increased $\mathrm{O}_{2}$ levels, recent studies have demonstrated that decreased $\mathrm{O}_{2}$ concentrations also trigger increased mitochondrial ROS production (36). These findings represent a paradigm shift in our understanding of oxygen homeostasis, in which cellular energy metabolism is modulated primarily to maintain redox homeostasis. This conclusion was supported by the finding that HIF-1 also modulates respiratory chain function in cancer cells by orchestrating a subunit switch (from COX4I1 to COX4I2) in cytochrome c oxidase that may increase the efficiency of electron transfer to $\mathrm{O}_{2}$ and allow continued respiration without increased ROS levels when $\mathrm{O}_{2}$ availability is modestly reduced (37).

The role of HIF-1 in mediating survival under hypoxic conditions is both cell type/tissue specific and context specific. For example, in mice exposed to $10 \% \mathrm{O}_{2}$, Cox $4 i 2$ mRNA expression was increased in liver and lung, but not in brain, heart, or kidney (37). In an IL-3-responsive cell line, HIF-1 $\alpha$ was required for survival under hypoxic conditions in the presence, but not absence, of IL-3, which was attributed to a requirement for HIF-dependent glycolysis in IL-3-stimulated cells (38).

HIFs also transactivate genes encoding microRNAs (miRs), which are small RNAs that bind to mRNAs in a sequence-specific manner to either inhibit their translation or induce their degradation (39). miR-210 expression is induced in response to hypoxia in many cell types (40). Among the targets of miR-210 that are downregulated in hypoxic cells are mRNAs encoded by the ISCU gene, which encodes an iron-sulfur cluster assembly enzyme that is required for the activity of mitochondrial electron transport complex I $(41,42)$. This represents another molecular strategy by which hypoxic cells decrease oxidative metabolism.

ROS levels vary widely in cancer cells, with high ROS levels often associated with cell proliferation (43), whereas low ROS levels 


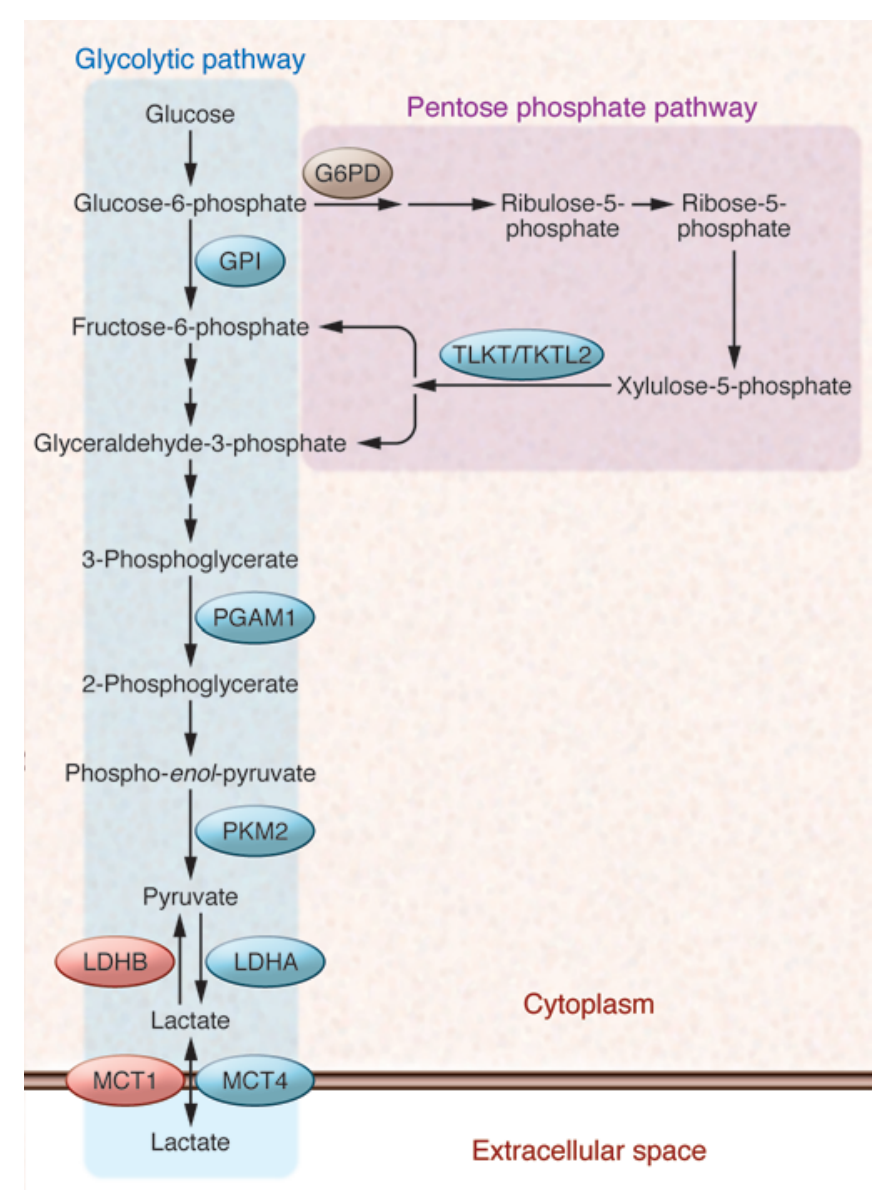

have been associated with resistance to chemotherapy (44) and the stem cell phenotype $(45,46)$. Thus, it is not clear to what extent decreased ROS production is utilized for cancer cell survival. Data from PC3 prostate cancer cells and P493 lymphoid cells indicate that increased ROS levels drive HIF- $1 \alpha$ expression in these cells and that antioxidants such as ascorbate or $\mathrm{N}$-acetylcysteine reduce HIF- $1 \alpha$ levels and tumor growth (47). Prolyl hydroxylases contain in their catalytic centers $\mathrm{Fe}(\mathrm{II})$, which is oxidized to $\mathrm{Fe}(\mathrm{III})$ during the reaction, inactivating the enzyme until it is reduced to $\mathrm{Fe}(\mathrm{II})$, which is facilitated by antioxidants, thereby promoting hydroxylation-dependent degradation of HIF- $1 \alpha$.

$p H$ regulation. Cancer cells decrease extracellular $\mathrm{pH}$ by activation of a battery of HIF-1 target genes encoding plasma membrane proteins (48). SLC16A3 encodes monocarboxylate transporter 4 (MCT4), which allows efflux of lactate out of cancer cells (49). SLC9A1 encodes sodium-hydrogen exchanger 1 (NHE1), which actively pumps $\mathrm{H}^{+}$ions into the extracellular space (50). CAR9 encodes carbonic anhydrase 9 (CA9), which converts $\mathrm{CO}_{2}+\mathrm{H}_{2} \mathrm{O}$ to $\mathrm{H}^{+}+\mathrm{HCO}_{3}{ }^{-}$and, acting in concert with $\mathrm{HCO}_{3}{ }^{-}$transporters, contributes to net efflux of $\mathrm{H}^{+}$from cancer cells (51). The consequences of MCT4, NHE1, and CA9 activity are intracellular alkalinization, which promotes proliferation, and extracellular acidification, which promotes invasion (52).

It is important to note that the cancer microenvironment is metabolically heterogeneous: cells adjacent to functional blood vessels are well oxygenated and more likely to utilize oxidative metabolism, whereas cells far from blood vessels are poorly oxygenated

\section{Figure 3}

Glucose flux through the glycolytic (Embden-Meyerhof) pathway and pentose phosphate pathway (PPP). G6PD shunts glucose-6-phosphate away from glycolysis and into the oxidative arm of the PPP. Transketolase (TKT) and transketolase-like 2 (TKTL2) shunt xylulose-5phosphate back into the glycolytic pathway. Only those glycolytic enzymes that are discussed specifically in the text are shown: glucose phosphate isomerase (GPI), PGAM1, PKM2, and LDHA and LDHB. Also shown are MCT4 and MCT1, which transport lactate out of and into cells, respectively.

and dependent upon glycolysis. In hypoxic cells, HIF-1 mediates increased expression of glucose transporters, glycolytic enzymes including LDHA, and MCT4. These changes in gene expression lead to increased glucose uptake, conversion to lactate, and extrusion of lactate from the cell (Figure 3). In contrast, oxygenated cells express MCT1 and LDHB, which mediate lactate uptake and conversion to pyruvate for oxidative metabolism. Disruption of this metabolic symbiosis by treatment of tumor-bearing mice with $\alpha$-cyano-4-hydroxycinnamate inhibited MCT1 activity such that aerobic cells were forced to take up glucose rather than lactate, leading to glucose depletion and the death of hypoxic cancer cells (53).

Fatty acid synthesis. The increased expression of BNIP3, LDHA, and PDK1 shunts pyruvate away from the mitochondria and reduces flux through the TCA pathway, the generation of NADH and $\mathrm{FADH}_{2}$ that feed the electron transport chain, and thus ROS generation under hypoxic conditions. However, reduced acCoA levels present a dilemma for those cells that continue to proliferate under hypoxic conditions with respect to fatty acid synthesis, which is normally dependent on glucose-derived acCoA that is converted to citrate, shuttled to the cytoplasm, and converted to acCoA by ATP citrate lyase (Figure 2A). Instead, hypoxic cancer cells utilize glutamine to generate $\alpha$-ketoglutarate, which is converted to citrate by isocitrate dehydrogenase and aconitase (54, 55). The switch from oxidative glucose metabolism to reductive glutamine metabolism as the source of acCoA for lipid synthesis under hypoxic conditions (Figure 2B) is HIF dependent and has been attributed to shunting of glucose-derived pyruvate away from the mitochondria by PDK1 $(54,55)$.

Glycogen synthesis. Conversion of glucose to glycogen provides a means to store metabolic substrate in reserve. Hypoxia induces the expression of genes encoding the enzymes required to convert glucose to glycogen, including hexokinase (HK1 or HK2), phosphoglucomutase 1 (PGM1), UDP-glucose pyrophosphorylase (UGP2), glycogen synthase (GYS1), glycogen branching enzyme (GBE1), as well as the gene encoding PPP1R3C, which activates GYS1 and inhibits liver-type glycogen phosphorylase (PYGL), the enzyme that breaks down glycogen (Figure 4). HIF-1 transactivates these genes as determined by microarray and ChIP assays (11, 12, 29, 56-59). PYGL is expressed at high levels in many cancer cells, and knockdown of PYGL expression in U87 glioma cells increased glycogen accumulation but also induced senescence, which was associated with increased ROS levels (58). The inability to liberate glucose-1-phosphate from glycogen in PYGL-knockdown U87 cells may reduce flux through the pentose phosphate pathway (PPP), which generates the NADPH required for ROS scavenging and lipid synthesis as well as nucleotides required for DNA synthesis.

HIF-1 controls the expression of genes encoding the enzymes required for the conversion of glucose either to glycogen (as described above) or to lactate (via the glycolytic pathway), which 


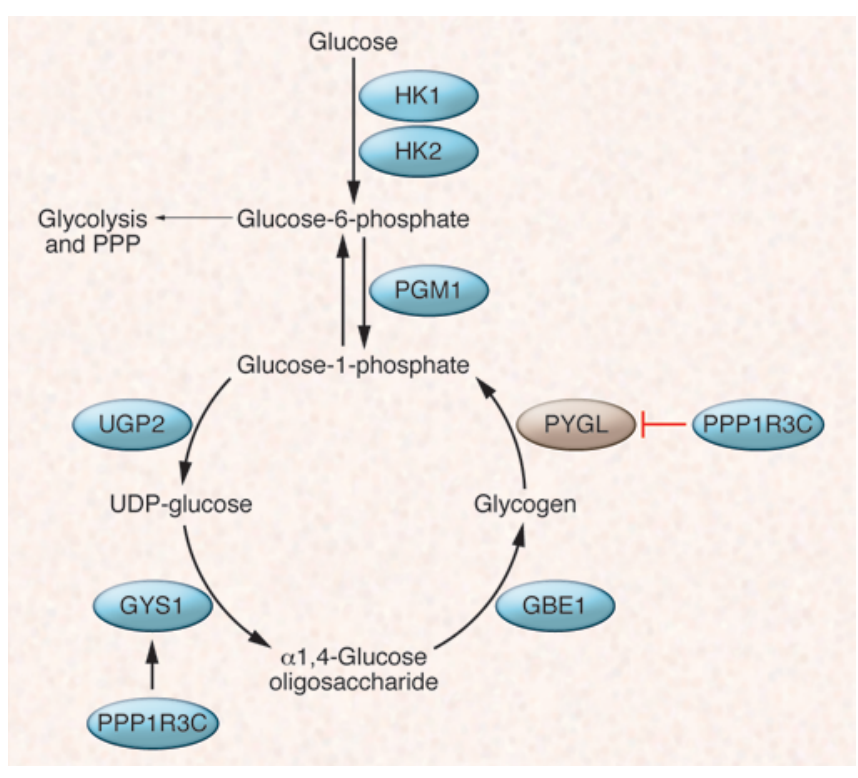

suggests that other factors may determine the relative flux through these two pathways in hypoxic cancer cells. Notably, depletion of glucosephosphate isomerase (GPI) or phosphoglycerate mutase 1 (PGAM1) (Figure 3) by RNA interference also induces senescence (60), indicating the critical role of glucose metabolism in maintaining proliferation. PGAM1 activity is required to maintain a proper balance between glycolysis and biosynthesis (via the PPP) for proliferation (61).

Multifunctional role of pyruvate kinase M2. HIF-1 activates the expression of pyruvate kinase M2 (PKM2), which encodes pyruvate kinase isoforms PKM1 and PKM2 by alternative splicing that includes sequences encoded by exon 9 or exon 10, respectively, which differ at 23 of 56 amino acids. In cancer cells, the expression of splicing factors favors the generation of PKM2 mRNA (62-65). PKM2 promotes tumor growth (66) by a complex and growing list of activities. First and foremost, PKM2 functions as a pyruvate kinase in glycolysis (Figure 3) but, in contrast to PKM1, its activity is regulated by allosteric interactions with phosphopeptides (67) and intermediary metabolites $(68,69)$ as well as by phosphorylation, acetylation, and oxidation (70-72). Second, PKM2 functions as a transcriptional coactivator for OCT 4 , HIF- $1 \alpha$ and HIF- $2 \alpha$, and $\beta$-catenin (73-75). HIF-1 activates transcription of PKM2, the protein product of which interacts with HIF-1 $\alpha$ to stimulate coactivator recruitment, chromatin binding, and transcriptional activation (74). This feed-forward loop increases expression of HIF-1 target genes encoding proteins that mediate the metabolic reprogramming of cancer cells (e.g., LDHA and PDK1), angiogenesis (e.g., VEGF), and other aspects of cancer progression. Finally, PKM2 functions as a protein kinase that phosphorylates STAT3, thereby increasing its transcriptional activity (76).

\section{Cancer-associated mutations alter metabolism in an HIF- dependent manner}

One of the consequences of many key genetic alterations in cancer cells is an increase in HIF activity (15). For example, PTEN or p53 loss of function leads to increased HIF-1 $\alpha$ synthesis and stability, respectively (77-79). Activation of tyrosine kinases (e.g., EGFR and HER2 in prostate and breast cancer, respectively) also increases HIF-1 $\alpha$ syn-

\section{Figure 4}

Glycogen synthesis and glycogenolysis. PGM1 shunts glucose-6phosphate away from glycolysis for use in glycogen synthesis, which requires the activity of three enzymes: UGP2, GYS1, and GBE1. Glucose-1-phosphate is released from glycogen (glycogenolysis) by PYGL. GYS1 and PYGL are positively and negatively regulated by protein phosphatase 1 regulatory subunit 3C (PPP1R3C).

thesis as a result of PI3K and AKT signaling that stimulates mTOR activity, which stimulates translation of HIF1A mRNA into protein by phosphorylation of eIF4E binding protein $(78,80)$. mTOR has also been reported to stimulate HIF-1 $\alpha$ stabilization and transactivation domain function (81). Studies of the Rous sarcoma virus (RSV) four decades ago revealed that one of the earliest changes in the phenotype of RSV-infected cells was an increased rate of glycolysis that was dependent upon the activity of the V-SRC oncoprotein (82-84), and $V$-SRC was the first oncogene shown to induce HIF-1 transcriptional activity, leading to increased expression of genes encoding glycolytic enzymes under nonhypoxic conditions (85).

The genetic alteration that most dramatically increases HIF activity is VHL loss of function, which is the hallmark of the clear cell type of renal cell carcinoma (86). In the absence of VHL, HIF$1 \alpha$ is not subjected to $\mathrm{O}_{2}$-dependent ubiquitylation and degradation (87), and increased HIF transcriptional activity is the earliest detectable sign of VHL loss of function in renal tubular cells (88). Loss of VHL resulted in an HIF-dependent, but $\mathrm{O}_{2}$-independent, switch from oxidative to glycolytic metabolism in RCC4 and RCC10 human renal carcinoma cells that dramatically reduced $\mathrm{O}_{2}$ consumption and respiration (89). VHL loss of function also led to HIF-dependent inhibition of PPAR $\gamma$ coactivator $1 \beta$ (PGC-1 $\beta$ ) expression and decreased mitochondrial biogenesis. All of these changes were reversed by forced expression of VHL or short-hairpin RNAs targeting HIF- $1 \alpha$ and HIF- $2 \alpha$ (89). These findings provided a definitive molecular basis for the observation first made by Otto Warburg that cancer cells manifest increased rates of lactate production under aerobic conditions (90). The transcription of genes encoding glycolytic enzymes is regulated in an HIF-1 $\alpha-$ dependent, but an HIF- $2 \alpha$-independent, manner in virtually all cell types except VHL-null renal carcinoma cells, in which HIF- $2 \alpha$ acquires this function by unknown mechanisms.

In addition to reprogramming glucose metabolism, VHL loss of function in renal carcinoma cells also leads to the switch from oxidative glucose metabolism to reductive glutamine metabolism as a source of acCoA for fatty acid synthesis that is described above as a response to hypoxia (Figure 2), but in VHL-null renal carcinoma cells this switch occurs in an $\mathrm{O}_{2}$-independent manner (55).

Whereas mutations in VHL directly block the ubiquitination of HIF- $\alpha$ subunits, mutations in the genes encoding succinate dehydrogenase (SDH) and fumarate hydratase (FH) block ubiquitination indirectly by increasing the concentration of TCA cycle intermediates that inhibit prolyl hydroxylase activity (91-93). In either case, these mutations increase HIF activity under nonhypoxic conditions, and this state is sometimes referred to as "pseudo-hypoxia," a term that should be discouraged because there are many cellular responses to hypoxia that are not regulated by VHL, PHD, or HIF activity. In contrast to the effect of loss-of-function mutations in genes encoding $\mathrm{SDH}$ or $\mathrm{FH}$, oncogenic missense mutations in the IDH1 or IDH2 gene encoding isocitrate dehydrogenase alter the catalytic activity of the enzyme such that it con- 
verts $\alpha$-ketoglutarate into D-2-hydroxyglutarate, which stimulates prolyl hydroxylase activity and results in decreased HIF- $\alpha$ protein levels (94), which contrasts with earlier reports of increased HIF- $\alpha$ levels in IDH-mutant cells (95). The effects of D-2-hydroxyglutarate as an oncometabolite may be due in part to its inhibition of the 5'-methylcytosine hydroxylase TET2 (96).

Although VHL-deficient renal cell carcinoma provides the most striking example of HIF-dependent metabolic reprogramming, many other genetic alterations appear to reprogram metabolism in a HIF-dependent manner. Chronic myeloid leukemia (CML) is characterized by the Philadelphia chromosome and expression of the transforming BCR-ABL fusion protein, which is targeted therapeutically by the tyrosine kinase inhibitor imatinib, although many patients eventually develop resistance (97). BCR-ABL signaling to mTOR leads to increased HIF- $1 \alpha$ protein levels and HIF-1 transcriptional activity in CML cells (98), and HIF-1 $\alpha$ is required for the maintenance of CML stem cells (99). When BCR-ABL-transformed cells were cultured in the presence of imatinib, the surviving cells expressed BCR-ABL and HIF-1 $\alpha$ at higher levels (100). Increased HIF-1 activity in resistant cells was associated with a shift of glucose flux from the TCA cycle to glycolysis. However, glucose flux also shifted from the oxidative arm of the PPP, which is initiated by glucose-6-phosphate dehydrogenase (G6PD), to the non-oxidative arm due to HIF-1-dependent expression of TKT and TKTL2, which encode transketolase (100). The PPP, a complicated collection of highly interconnected enzymatic reactions, is presented in a highly simplified manner in Figure 3 to illustrate that activation of TKT and TKTL2 expression provides a measure of metabolic flexibility by allowing return of intermediates to the glycolytic pathway. Treatment with oxythiamine, an inhibitor of TKT/TKTL2, led to increased sensitivity to imatinib in vivo (100). As in the cases of PYGL knockdown and MCT1 inhibition described above, these results indicate that pharmacologic strategies designed to limit metabolic flexibility may have anticancer effects.

\section{Clinical implications}

Translational efforts are underway to devise novel anticancer strategies involving inhibition of the metabolic enzymes that are induced by HIF. An example is the use of dichloroacetate (DCA), which is an inhibitor of PDK activity (101). DCA has been used to treat congenital lactic acidosis in children with mitochondrial disorders (102). When DCA was administered in the drinking water of athymic rats bearing subcutaneous xenografts of A549 human lung cancer cells, xenograft growth was significantly inhibited (103). Inhibition of PDK activity by DCA has been reported to inhibit HIF-1 transcriptional activity by increasing $\alpha$-ketoglutarate levels, thereby leading to increased prolyl hydroxylase activity (104). In a clinical study of three patients with glioblastoma multiforme, comparison of tumor tissue before and after DCA treatment revealed increased PDH activity, decreased proliferation, increased apoptosis, decreased HIF-1 $\alpha$ levels, and decreased angiogenesis after therapy (105). A clinical trial is underway to study the safety and efficacy of DCA in patients with glioblastoma and other recurrent brain tumors (ClinicalTrials.gov identifier: NCT01111097). An alternative, but not mutually exclusive, strategy is to inhibit HIF activity as a means of altering multiple metabolic pathways as well as blocking the expression of genes encoding proteins that contribute to angiogenesis, invasion, and metastasis (24). Digoxin decreases HIF$1 \alpha$ protein levels and inhibits primary tumor growth, lymphatic metastasis to axillary lymph nodes, and hematogenous metastasis to the lungs in an orthotopic model of breast cancer (106-109), and a study is underway to determine whether digoxin inhibits HIF-1 activity in human breast cancer (ClinicalTrials.gov identifier: NCT01763931). Preclinical data suggest that both metabolic inhibitors such as DCA (110) and HIF inhibitors (111) may improve the currently limited efficacy of angiogenesis inhibitors such as the anti-VEGF antibody bevacizumab.

Several strategies are being employed in an effort to identify subsets of cancers that will respond to inhibition of particular metabolic pathways, including the use of algorithms that infer metabolic phenotypes from RNA/protein expression data (112), metabolomic profiling techniques (113), stable isotope-based metabolic analysis $(114,115)$, and functional genomic strategies (116). Although the complexity and interconnectedness of metabolic pathways suggest that targeting a single metabolic pathway may have limited anticancer effects, preclinical studies suggest that by limiting metabolic flexibility, inhibitors of metabolic enzymes or master regulators such as HIF-1 may improve the response to cytotoxic chemotherapy and compounds that target signal transduction pathways.

\section{Acknowledgments}

Work in the author's laboratory is supported by the C. Michael Armstrong Professorship at Johns Hopkins University School of Medicine; funds from the Johns Hopkins Institute for Cell Engineering; and grants/contracts from the American Cancer Society, Department of Defense (W81XWH-12-1-0464), NIH (U54CA143868 and HHS-N268201000032C), and Susan G. Komen for the Cure Research Programs.

Address correspondence to: Gregg L. Semenza, Miller Research Building, Suite 671, 733 N. Broadway, Baltimore, Maryland 21205, USA. Phone: 410.955.1619; Fax: 443.287.5618; E-mail: gsemenza@jhmi.edu.
1. Wang GL, Semenza GL. Purification and characterization of hypoxia-inducible factor 1. J Biol Chem. 1995;270(3):1230-1237.

2. Wang GL, Jiang BH, Rue EA, Semenza GL. Hypoxia-inducible factor 1 is a basic-helix-loop-helix-PAS heterodimer regulated by cellular $\mathrm{O}_{2}$ tension. Proc Natl Acad Sci U S A. 1995;92(12):5510-5514.

3. Semenza GL, et al. Hypoxia response elements in the aldolase A, enolase 1 , and lactate dehydrogenase A gene promoters contain essential binding sites for hypoxia-inducible factor 1. J Biol Chem. 1996;271(51):32529-32537.

4. Kaelin WG Jr, Ratcliffe PJ. Oxygen sensing by metazoans: the central role of the HIF hydroxylase pathway. Mol Cell. 2008;30(4):393-402.
5. Tian H, McKnight SL, Russell DW. Endothelial PAS domain protein 1 (EPAS1), a transcription factor selectively expressed in endothelial cells. Genes Dev. 1997;11(1):72-82.

6. Wiesener MS, et al. Induction of endothelial PAS domain protein-1 by hypoxia: characterization and comparison with hypoxia-inducible factor- $1 \alpha$. Blood. 1998;92(7):2260-2268.

7. Hampton-Smith RJ, Peet DJ. From polyps to people: a highly familiar response to hypoxia. Ann NY Acad Sci. 2009;1177:19-29.

8. Loenarz C, et al. The hypoxia-inducible transcription factor pathway regulates oxygen sensing in the simplest animal, Trichoplax adhaerens. EMBO Rep. 2011;12(1):63-70.
9. Zhong $\mathrm{H}$, et al. Overexpression of hypoxia-inducible factor $1 \alpha$ in common human cancers and their metastases. Cancer Res. 1999;59(22):5830-5835.

10. Talks KL, et al. The expression and distribution of the hypoxia-inducible factors HIF- $1 \alpha$ and HIF- $2 \alpha$ in normal human tissues, cancers, and tumor-associated macrophages. Am J Pathol. 2000;157(2):411-421.

11. Mole DR, et al. Genome-wide association of hypoxia-inducible factor (HIF) $-1 \alpha$ and HIF$2 \alpha$ DNA binding with expression profiling of hypoxia-inducible transcripts. J Biol Chem. 2009; 284(25):16767-16775.

12. Xia X, et al. Integrative analysis of HIF binding and transactivation reveals its role in maintaining histone methylation homeostasis. Proc Natl Acad Sci 
US A. 2009;106(11):4260-4265.

13. Schödel J, Oikonomopoulos S, Ragoussis J, Pugh CW, Ratcliffe PJ, Mole DR. High-resolution genome-wide mapping of HIF-binding sites by ChIP-seq. Blood. 2011;117(23):e207-e217.

14. Vaupel P, Mayer A, Höckel M. Tumor hypoxia and malignant progression. Meth Enzymol. 2004;381:335-354

15. Semenza GL. Defining the role of hypoxia-inducible factor 1 in cancer biology and therapeutics. Oncogene. 2010;29(5):625-634.

16. Kroeger N, et al. Deletions of chromosomes $3 p$ and $14 \mathrm{q}$ molecularly subclassify clear cell renal cell carcinoma. Cancer. 2013;119(8):1547-1554.

17. Imamura T, et al. HIF- $1 \alpha$ and HIF- $2 \alpha$ have divergent roles in colon cancer. Int $J$ Cancer. 2009;124(4):763-771.

18. Keith B, Johnson RS, Simon MC. HIF- $1 \alpha$ and HIF$2 \alpha$ : sibling rivalry in hypoxic tumor growth and progression. Nat Rev Cancer. 2011;12(1):9-22

19. Mole DR, et al. Genome-wide association of hypoxia-inducible factor (HIF)- $1 \alpha$ and HIF$2 \alpha$ DNA binding with expression profiling of hypoxia-inducible transcripts. J Biol Chem. 2009;284(25):16767-16775.

20. Lau KW, Tian YM, Raval RR, Ratcliffe PJ, Pugh $\mathrm{CW}$. Target gene selectivity of hypoxia-inducible factor- $\alpha$ in renal cancer cells is conveyed by post-DNA-binding mechanisms. Br $\mathrm{J}$ Cancer. 2007;96(8):1284-1292.

21. Forsythe JA, et al. Activation of vascular endothelial growth factor gene transcription by hypoxia-inducible factor 1. Mol Cell Biol. 1996;16(9):4604-4613.

22. Rey S, Semenza GL. Hypoxia-inducible factor-1-dependent mechanisms of vascularization and vascular remodelling. Cardiovasc Res. 2010;86(2):236-242.

23. Carmeliet P, Jain RK. Principles and mechanisms of vessel normalization for cancer and other angiogenic diseases. Nat Rev Drug Discov. 2011;10(6):417-427.

24. Semenza GL. Molecular mechanisms mediating metastasis of hypoxic breast cancer cells. Trends $\mathrm{Mol}$ Med. 2012;18(9):534-543.

25. Koshiji M, Kageyama Y, Pete EA, Horikawa I, Barrett JC, Huang LE. HIF- $1 \alpha$ induces cell cycle arrest by functionally counteracting Myc. $E M B O J$. 2004;23(9):1949-1956

26. Kaidi A, Williams AC, Paraskeva C. Interaction between $\beta$-catenin and HIF-1 promotes cellular adaptation to hypoxia. Nat Cell Biol. 2007;9(2):210-217.

27. Corn PG, et al. Mxi1 is induced by hypoxia in a HIF-1-dependent manner and protects cells from c-Myc-induced apoptosis. Cancer Biol Ther. 2005;4(11):1285-1294

28. Hubbi ME, et al. A nontranscriptional role for HIF$1 \alpha$ as a direct inhibitor of DNA replication. Sci Signal. 2013;6(262):ra10

29. Iyer NV, et al. Cellular and developmental control of $\mathrm{O}_{2}$ homeostasis by hypoxia-inducible factor $1 \alpha$. Genes Dev. 1998;12(2):149-162.

30. Kim JW, Tchernyshyov I, Semenza GL, Dang CV. HIF-1-mediated expression of pyruvate dehydrogenase kinase: a metabolic switch required for cellular adaptation to hypoxia. Cell Metab. 2006;3(3):177-185

31. Zhang H, et al. Mitochondrial autophagy is an HIF-1-dependent adaptive metabolic response to hypoxia. J Biol Chem. 2008;283(16):10892-10903.

32. Papandreou I, Cairns RA, Fontana L, Lim AL, Denko NC. HIF-1 mediates adaptation to hypoxia by actively downregulating mitochondrial oxygen consumption. Cell Metab. 2006;3(3):187-197.

33. Sowter HM, Ratcliffe PJ, Watson P, Greenberg AH, Harris AL. HIF-1-dependent regulation of hypoxic induction of the cell death factors BNIP3 and NIX in human tumors. Cancer Res. 2001;61(18):6669-6673.

34. Lu CW, Lin SC, Chen KF, Lai YY, Tsai SJ. Induction of pyruvate dehydrogenase kinase- 3 by hypoxia-inducible factor- 1 promotes metabolic switch and drug resistance. J Biol Chem. 2008;283(42):28106-28114.

35 . Bellot G, et al. Hypoxia-induced autophagy is mediated through hypoxia-inducible factor induction of BNIP3 and BNIP3L via their BH3 domains. Mol Cell Biol. 2009;29(10):2570-2581.

36. Chandel NS, et al. Reactive oxygen species generated at mitochondrial complex III stabilize hypoxia-inducible factor- $1 \alpha$ during hypoxia: a mechanism of $\mathrm{O}_{2}$ sensing. J Biol Chem. 2000;275(33):25130-25138.

37. Fukuda R, Zhang H, Kim JW, Shimoda L, Dang CV, Semenza GL. HIF-1 regulates cytochrome oxidase subunits to optimize efficiency of respiration in hypoxic cells. Cell. 2007;129(1):111-122.

38. Lum JJ, et al. The transcription factor HIF- $1 \alpha$ plays a critical role in the growth factor-dependent regulation of both aerobic and anaerobic glycolysis. Genes Dev. 2007;21(9):1037-1049.

39. Ivan M, Harris AL, Martelli F, Kulshreshtha R. Hypoxia response and microRNAs: no longer two separate worlds. J Cell Mol Med. 2008; 12(5A):1426-1431.

40. Devlin C, Greco S, Martelli F, Ivan M. miR-210: More than a silent player in hypoxia. IUBMB Life. 2011;63(2):94-100.

41. Chan SY, Zhang YY, Hemann C, Mahoney CE, Zweier JL, Loscalzo J. MicroRNA-210 controls mitochondrial metabolism during hypoxia by repressing the iron-sulfur cluster assembly proteins ISCU1/2. Cell Metab. 2009;10(4):273-284

42. Favaro E, et al. MicroRNA-210 regulates mitochondrial free radical response to hypoxia and krebs cycle in cancer cells by targeting iron sulfur cluster protein ISCU. PLoS One. 2010;5(4):e10345.

43. Finkel T. Signal transduction by reactive oxygen species. J Cell Biol. 2011;194(1):7-15.

44. Cuezva JM, et al. The bioenergetic signature of cancer: a marker of tumor progression. Cancer Res. 2002;62(22):6674-6681

45. Suda T, Takubo K, Semenza GL. Metabolic regulation of hematopoietic stem cells in the hypoxic niche. Cell Stem Cell. 2011;9(4):298-310.

46. Lagadinou ED, et al. BCL-2 inhibition targets oxidative phosphorylation and selectively eradicates quiescent human leukemia stem cells. Cell Stem Cell. 2013;12(3):329-341.

47. Gao P, et al. HIF-dependent antitumorigenic effect of antioxidants in vivo. Cancer Cell. 2007;12(3):230-238.

48. Semenza GL. Regulation of cancer cell metabolism by hypoxia-inducible factor 1. Semin Cancer Biol. 2009;19(1):12-16.

49. Pinheiro C, Longatto-Filho A, Azevedo-Silva J, Casal M, Schmitt FC, Baltazar F. Role of monocarboxylate transporters in human cancers: state of the art. J Bioenerg Biomembr. 2012;44(1):127-139.

50. Chiche J, Brahimi-Horn MC, Pouysségur J. Tumor hypoxia induces a metabolic shift causing acidosis: a common feature in cancer. J Cell Mol Med. 2010;14(4):771-794.

51. Swietach P, Wigfield S, Cobden P, Supuran CT, Harris AL, Vaughan-Jones RD. Tumor-associated carbonic anhydrase 9 spatially coordinates intracellular $\mathrm{pH}$ in three-dimensional multicellular growths. J Biol Chem. 2008;283(29):20473-20483.

52. Gatenby RA, et al. Cellular adaptations to hypoxia and acidosis during somatic evolution of breast cancer. Br J Cancer. 2007;97(5):646-653.

53. Sonveaux $\mathrm{P}$, et al. Targeting lactate-fueled respiration selectively kills hypoxic tumor cells in mice. J Clin Invest. 2008;118(12):3930-3942.

54 . Wise DR, et al. Hypoxia promotes isocitrate dehydrogenase-dependent carboxylation of $\alpha$-ketoglutarate to citrate to support cell growth and viability. Proc Natl Acad Sci U S A. 2011;108(49):19611-19616.

55. Metallo CM, et al. Reductive glutamine metabolism by IDH1 mediates lipogenesis under hypoxia. Nature. 2011;481(7381):380-384.

56. Manalo DJ, et al. Transcriptional regulation of vascular endothelial cell responses to hypoxia by HIF-1.
Blood. 2005;105(2):659-669.

57 . Pescador $\mathrm{N}$, et al. Hypoxia promotes glycogen accumulation through hypoxia inducible factor (HIF)mediated induction of glycogen synthase 1. PLoS One. 2010;5(3):e9644

58. Favaro E, et al. Glucose utilization via glycogen phosphorylase sustains proliferation and prevents premature senescence in cancer cells. Cell Metab. 2012;16(6):751-764.

59. Mimura I, et al. Dynamic change of chromatin conformation in response to hypoxia enhances the expression of GLUT3 (SLC2A3) by cooperative interaction of hypoxia-inducible factor 1 and KDM3A. Mol Cell Biol. 2012;32(15):3018-3032.

60 . Kondoh $\mathrm{H}$, et al. Glycolytic enzymes can modulate cellular life span. Cancer Res. 2005;65(1):177-185.

61. Hitosugi T, et al. Phosphoglycerate mutase 1 coordinates glycolysis and biosynthesis to promote tumor growth. Cancer Cell. 2012;22(5):585-600.

62. Clower $\mathrm{CV}$, et al. The alternative splicing repressors hnRNP A1/A2 and PTB influence pyruvate kinase isoform expression and cell metabolism. Proc Natl Acad Sci U S A. 2010;107(5):1894-1899.

63. David CJ, Chen M, Assanah M, Canoll P, Manley JL. HnRNP proteins controlled by c-Myc deregulate pyruvate kinase mRNA splicing in cancer. Nature. 2010;463(7279):364-368.

64. Chen M, David CJ, Manley JL. Concentration-dependent control of pyruvate kinase M mutually exclusive splicing by hnRNP proteins. Nat Struct Mol Biol. 2012;19(3):346-354.

65 . Wang $Z$, et al. Exon-centric regulation of pyruvate kinase $M$ alternative splicing via mutually exclusive exons. J Mol Cell Biol. 2012;4(2):79-87.

66. Christofk HR, et al. The M2 splice isoform of pyruvate kinase is important for cancer metabolism and tumor growth. Nature. 2008;452(7184):230-233.

67. Christofk HR, Vander Heiden MG, Wu N, Asara JM, Cantley LC. Pyruvate kinase M2 is a phosphotyrosine-binding protein. Nature. 2008; 452(7184):181-186.

68. Chaneton B, et al. Serine is a natural ligand and allosteric activator of pyruvate kinase M2. Nature. 2012;491(7424):458-462.

69. Keller KE, Tan IS, Lee YS. SAICAR stimulates pyruvate kinase isoform M2 and promotes cancer cell survival in glucose-limited conditions. Science. 2012;338(6110):1069-1072.

70. Hitosugi T, et al. Tyrosine phosphorylation inhibits PKM2 to promote the Warburg effect and tumor growth. Sci Signal. 2009;2(97):ra73.

71. Lv L, et al. Acetylation targets the M2 isoform of pyruvate kinase for degradation through chaperone-mediated autophagy and promotes tumor growth. Mol Cell. 2011;42(6):719-730.

72. Anastasiou D, et al. Inhibition of pyruvate kinase M2 by reactive oxygen species contributes to cellular antioxidant responses. Science. 2011; 334(6060):1278-1283

73. Lee J, Kim HK, Han YM, Kim J. Pyruvate kinase isozyme type M2 (PKM2) interacts and cooperates with Oct- 4 in regulating transcription. Int J Biochem Cell Biol. 2008;40(5):1043-1054.

74. Luo W, et al. Pyruvate kinase M2 is a PHD3-stimulated coactivator for hypoxia-inducible factor 1 . Cell. 2011;145(5):732-744.

75. Yang W, et al. Nuclear PKM2 regulates $\beta$-catenin transactivation upon EGFR activation. Nature. 2011;480(7375):118-122.

76. Gao X, Wang H, Yang JJ, Liu X, Liu ZR. Pyruvate kinase $\mathrm{M} 2$ regulates gene transcription by acting as a protein kinase. Mol Cell. 2012;45(5):598-609.

77. Ravi R, et al. Regulation of tumor angiogenesis by p53-induced degradation of hypoxia-inducible factor $1 \alpha$. Genes Dev. 2000;14(1):34-44.

78. Zhong $\mathrm{H}$, et al. Modulation of hypoxia-inducible factor $1 \alpha$ expression by the epidermal growth factor/phosphatidylinositol 3-kinase/PTEN/AKT/ 
FRAP pathway in human prostate cancer cells: implications for tumor angiogenesis and therapeutics. Cancer Res. 2000;60(6):1541-1545.

79. Zundel W, et al. Loss of PTEN facilitates HIF-1-mediated gene expression. Genes Dev. 2000;14(4):391-396.

80. Laughner E, Taghavi P, Chiles K, Mahon PC, Semenza GL. HER2 (neu) signaling increases the rate of hypoxia-inducible factor $1 \alpha(\mathrm{HIF}-1 \alpha)$ synthesis: novel mechanism for HIF-1-mediated vascular endothelial growth factor expression. Mol Cell Biol. 2001;21(12):3995-4004.

81. Hudson CC, et al. Regulation of hypoxia-inducible factor $1 \alpha$ expression and function by the mammalian target of rapamycin. Mol Cell Biol. 2002;22(20):7004-7014.

82. Steck TL, Kaufman S, Bader JP. Glycolysis in chick embryo cell cultures transformed by Rous sarcoma virus. Cancer Res. 1968;28(8):1611-1619.

83. Singh VN, Singh M, August JT, Horecker BL. Alterations in glucose metabolism in chick-embryo cells transformed by Rous sarcoma virus: intracellular levels of glycolytic intermediates. Proc Natl Acad Sci U S A. 1974;71(10):4129-4132.

84. Carroll RC, Ash JF, Vogt PK, Singer SJ. Reversion of transformed glycolysis to normal by inhibition of protein synthesis in rat kidney cells infected with temperature-sensitive mutant of Rous sarcoma virus. Proc Natl Acad Sci U S A. 1978; 75(10):5015-5019.

85. Jiang BH, Agani F, Passaniti A, Semenza GL. V-SRC induces expression of hypoxia-inducible factor 1 (HIF-1) and transcription of genes encoding vascular endothelial growth factor and enolase 1 : involvement of HIF-1 in tumor progression. Cancer Res. 1997;57(23):5328-5335.

86. Kaelin WG Jr. The von Hippel-Lindau tumour suppressor protein: $\mathrm{O}_{2}$ sensing and cancer. Nat Rev Cancer. 2008;8(11):865-873.

87. Maxwell $\mathrm{PH}$, et al. The tumor suppressor protein VHL targets hypoxia-inducible factors for oxygen-dependent proteolysis. Nature. 1999;399(6733):271-275.

88. Mandriota SJ, et al. HIF activation identifies early lesions in VHL kidneys: evidence for site-specific tumor suppressor function in the nephron. Cancer Cell. 2002;1(5):459-468

89. Zhang $\mathrm{H}$, et al. HIF-1 inhibits mitochondrial biogenesis and cellular respiration in VHL-deficient renal cell carcinoma by repression of C-MYC activ- ity. Cancer Cell. 2007;11(5):407-420.

90. Warburg O. Über den stoffwechsel der carcinomzelle. Klin Wochenschr. 1925;4:534-536.

91. Isaacs JS, et al. HIF overexpression correlates with biallelic loss of fumarate hydratase in renal cancer: novel role of fumarate in regulation of HIF stability. Cancer Cell. 2005;8(2):143-153.

92. Pollard PJ, et al. Accumulation of Krebs cycle intermediates and over-expression of HIF-1a in tumors which result from germline $\mathrm{FH}$ and SDH mutations. Hum Mol Genet. 2005;14(15):2231-2239.

93. Selak MA, et al. Succinate links TCA cycle dysfunction to oncogenesis by inhibiting HIF-a prolyl hydroxylase. Cancer Cell. 2005;7(1):77-85.

94. Koivunen P, et al. Transformation by the (R)-enantiomer of 2-hydroxyglutarate linked to EGLN activation. Nature. 2012;483(7390):484-488.

95. Zhao S, et al. Glioma-derived mutations in IDH1 dominantly inhibit IDH1 catalytic activity and induce HIF-1 $\alpha$. Science. 2009;324(5924):261-265.

96. Losman JA, et al. (R)-2-hydroxyglutarate is sufficient to promote leukemogenesis and its effects are reversible. Science. 2013;339(6127):1621-1625.

97. Sherbenou DW, Druker BJ. Applying the discovery of the Philadelphia chromosome. J Clin Invest. 2007;117(8):2067-2074.

98. Mayerhofer M, Valent P, Sperr WR, Griffin JD, Sillaber $\mathrm{C}$. BCR/ABL induces expression of vascular endothelial growth factor and its transcriptional activator, hypoxia inducible factor- $1 \alpha$, through a pathway involving phosphoinositide 3-kinase and the mammalian target of rapamycin. Blood. 2002;100(10):3767-3775.

99. Zhang H, Li H, Xi HS, Li S. HIF- $1 \alpha$ is required for survival maintenance of chronic myeloid leukemia stem cells. Blood. 2012;119(11):2595-2607.

100.Zhao F, et al. Imatinib resistance associated with BCR-ABL upregulation is dependent on HIF- $1 \alpha$ induced metabolic reprogramming. Oncogene. 2010;29(20):2962-2972.

101. Stacpoole PW. The pharmacology of dichloroacetate. Metabolism. 1989;38(11):1124-1144.

102. Stacpoole PW, et al. Controlled clinical trial of dichloroacetate for treatment of congenital lactic acidosis in children. Pediatrics. 2006;117(5):1519-1531.

103. Bonnet $\mathrm{S}$, et al. A mitochondria- $\mathrm{K}^{+}$channel axis is suppressed in cancer and its normalization promotes apoptosis and inhibits cancer growth. Cancer Cell. 2007;11(1):37-51.
104. Sutendra G, et al. Mitochondrial activation by inhibition of PDKII suppresses HIF- $1 \alpha$ signaling and angiogenesis in cancer. Oncogene. 2013;32(13):1638-1650.

105. Michelakis ED, et al. Metabolic modulation of glioblastoma with dichloroacetate. Sci Transl Med. 2010;2(31):31ra34.

106. Schito L, et al. Hypoxia-inducible factor 1-dependent expression of platelet-derived growth factor B promotes lymphatic metastasis of hypoxic breast cancer cells. Proc Natl Acad Sci U S A. 2012;109(40):E2707-E2716.

107. Wong CC, et al. Inhibitors of hypoxia-inducible factor 1 block breast cancer metastatic niche formation and lung metastasis. J Mol Med (Berl). 2012;90(7):803-815.

108.Zhang H, et al. HIF-1-dependent expression of angiopoietin-like 4 and L1CAM mediates vascular metastasis of hypoxic breast cancer cells to the lungs. Oncogene. 2012;31(14):1757-1770.

109. Chaturvedi $P$, et al. Hypoxia-inducible factor-dependent breast cancer-mesenchymal stem cell bidirectional signaling promotes metastasis. J Clin Invest. 2013;123(1):189-205.

110.Kumar K, et al. Dichloroacetate reverses the hypoxic adaptation to bevacizumab and enhances its antitumor effects in mouse xenografts. $J \mathrm{Mol}$ Med (Berl). 2013;91(6):749-758.

111. Rapisarda A, et al. Increased antitumor activity of bevacizumab in combination with hypoxia inducible factor-1 inhibition. Mol Cancer Ther. 2009;8(7):1867-1877.

112.Jerby L, et al. Metabolic associations of reduced proliferation and oxidative stress in advanced breast cancer. Cancer Res. 2012;72(22):5712-5720.

113.Lu X, Bennet B, Mu E, Rabinowitz J, Kang Y. Metabolomic changes accompanying transformation and acquisition of metastatic potential in a syngeneic mouse mammary tumor model. J Biol Chem. 2010;285(13):9317-9321.

114.Locasale JW, et al. Phosphoglycerate dehydrogenase diverts glycolytic flux and contributes to oncogenesis. Nat Genet. 2011;43(9):869-874.

115.Yuneva MO, et al. The metabolic profile of tumors depends on both the responsible genetic lesion and tissue type. Cell Metab. 2011;15(2):157-170.

116. Birsoy K, et al. MCT1-mediated transport of a toxic molecule is an effective strategy for targeting glycolytic tumors. Nat Genet. 2012;45(1):104-108. 